\title{
The age of onset and evolution of Braak tangle stage and Thal amyloid pathology of Alzheimer's disease in individuals with Down syndrome
}

\author{
Yvonne S. Davidson ${ }^{1}$, Andrew Robinson ${ }^{1}$, Vee P. Prasher ${ }^{2,3}$ and David M. A. Mann ${ }^{1 *}$ (D)
}

\begin{abstract}
While post mortem studies have identified the major cell types and functional systems affected in Alzheimer's disease (AD) the initial sites and molecular characteristics of pathology are still unclear. Because individuals with Down syndrome (DS) (trisomy 21) develop the full pathological changes of AD in a predictable way by the time they reach middle to late age, a study of the brains of such persons at different ages makes an ideal 'model system' in which the sites of earliest onset of pathology can be detected and the subsequent progression of changes be monitored. In the present study we have examined the brains of 56 individuals with DS ranging from new-born to 76 years for the presence of amyloid and tau pathology in key cortical and subcortical regions. Amyloid pathology was found to commence in the late teens to twenties as a deposition of diffuse plaques initially within the temporal neocortex, quickly involving other neocortical regions but only reaching subcortical regions and cerebellum by the late forties. Cerebral amyloid angiopathy did not regularly commence until after 45-50 years of age. Tau pathology usually commenced after 35 years of age, initially involving not only entorhinal areas and hippocampus but also subcortical regions such as locus caeruleus (LC) and dorsal raphe nucleus (DRN). Later, tau pathology spread throughout the neocortex reaching occipital lobes in most instances by mid-50 years of age. Such a pattern of spread is consistent with that seen in typical AD. We found no evidence that tau pathology might commence within the brain in DS before amyloid deposition had occurred, but there was limited data that suggests tau pathology in LC or DRN might predate that in entorhinal areas and hippocampus or at least be coincident.
\end{abstract}

Keywords: Alzheimer's disease, Down syndrome, Amyloid, Tau

\section{Introduction}

Alzheimer's disease (AD) is characterised pathologically by the abundant presence of deposits of amyloid $\beta$ protein $(\mathrm{A} \beta)$ in the brain parenchyma in the form of amyloid plaques, and in blood vessel walls as cerebral amyloid angiopathy (CAA), and neurofibrillary tangles (NFT) composed of hyperphosphorylated tau proteins $[19,35]$. While post mortem studies based on established cases of $\mathrm{AD}$ have identified the major cell types and functional systems affected by the disorder, the initial sites of pathology are

\footnotetext{
* Correspondence: david.mann@manchester.ac.uk

${ }^{1}$ Institute of Brain, Behaviour and Mental Health, Faculty of Medical and Human Sciences University of Manchester, Salford Royal Hospital, Stott Lane, Salford M6 8HD, England

Full list of author information is available at the end of the article
}

still unclear. According to the original and modified Braak stageing protocols $[4,7]$, the earliest sites of tau pathology lie within the entorhinal and transentorhinal cortex (stage I), spreading to hippocampus (stage II), temporal cortex (stage III) and eventually to other regions of cerebral cortex (stage IV), finally reaching visual association cortex (stage V) and primary visual cortex (stage VI). Conversely, the order of amyloid plaque deposition [46] commences within neocortical regions, particularly within the temporal lobe (phase 1), and spreads to involve allocortical regions such as the hippocampus and amygdala (phase 2), then into subcortical regions (phase 3), brain stem (phase 4) and finally cerebellum (phase 5).

However, this concept of the primacy and spread of tau pathology in AD has recently been questioned. From

(c) The Author(s). 2018 Open Access This article is distributed under the terms of the Creative Commons Attribution 4.0 International License (http://creativecommons.org/licenses/by/4.0/), which permits unrestricted use, distribution, and reproduction in any medium, provided you give appropriate credit to the original author(s) and the source, provide a link to the Creative Commons license, and indicate if changes were made. The Creative Commons Public Domain Dedication waiver (http://creativecommons.org/publicdomain/zero/1.0/) applies to the data made available in this article, unless otherwise stated. 
a study of 42 cognitively normal individuals under 30 years of age, Braak and Del Tredici suggested that the earliest site of tau pathology in AD might actually occur within certain subcortical nuclei, notably the locus caeruleus (LC), predating that within the entorhinal area [6]. Of these 42 individuals, deposits of $A \beta$ were present in only 1 person, a 17 -year old with Down Syndrome (DS), simultaneously challenging the concept of the amyloid cascade hypothesis of AD [14]. Another study by this same research group of 2332 unselected post mortems ranging from 1 to 100 years of age [8] found tau pathology in 58 cases, in the absence of $A \beta$ deposition in all but one instance (again the same case of DS as included in [6]). Tau pathology was essentially restricted to those individuals aged 30 years or less, and occurred in LC and certain other non-cortical nuclei such as the upper raphe in the brainstem and the nucleus basalis of Meynert in the basal forebrain, without there being any entorhinal/cortical involvement. These data were interpreted as providing evidence for there being stages in the pathological process of $\mathrm{AD}$ that predate those affecting entorhinal/transentorhinal areas, and that changes in these subcortical nuclei may represent the very earliest sites of damage to the brain in AD. Nevertheless, from these kinds of observational studies it is not possible to predict whether any of the affected individuals would have gone on to develop AD had they survived longer. Moreover, the lack of an (inducible) animal model that fully recapitulates the pathological process of $\mathrm{AD}$ precludes experimental studies of the time course of the development of AD pathology.

On the other hand, it has long been known that individuals with Down syndrome (DS) associated with an additional copy of chromosome 21 (trisomy 21) develop the full pathological changes of AD by the time they reach middle age, and sometimes as young as 40 years $[9,13,16,18,28-30,38,42,50]$. Hence, a study of the brains of such persons at different ages makes an ideal 'model system' in which the earliest sites of Alzheimer-type pathology can be observed and the subsequent progression of such changes be monitored. As with individuals with duplications in APP, DS is associated with an overexpression of APP protein, and therefore it might be argued that DS represents a model of $A \beta$ hyperproduction rather than AD per se. Nonetheless, it still remains closer in pathological terms to $\mathrm{AD}$ than any other human condition or experimental paradigm. In the present study we have examined the brains of 56 individuals with DS ranging from new-born to 76 years for the presence of amyloid and tau pathology in certain cortical and subcortical regions and have charted the onset and progression of these, and other, pathologies in each brain region with age, and in relationship to each other.

\section{Material and methods}

Serial wax sections were cut at $6 \mu \mathrm{m}$ thickness from formalin fixed tissue blocks from the brains of 56 individuals with DS (34 males, 22 females) ranging from new-born to 76 years (mean $48.0 \pm 20.2$ years). There was no significant difference in mean age between males and females (males, $48.3 \pm 20.7$ years; females, $47.4 \pm 19.8$ years). Thirteen of the 56 cases were drawn from the Manchester Brain Bank (MBB), 7 were obtained from Institute of Psychiatry (IOP) Brain Bank, London, 22 from the Thomas Willis Brain Bank (TWBB), Oxford, with the remaining 14 being obtained through Professor V P Prasher at University of Birmingham. All brains had been obtained at autopsy through appropriate consenting procedures with Local Ethical Committee approval. Selected case details are provided in Table 1. Details concerning karyotyping were only available for 13 individuals, but all 13 were a full trisomy 21 (Table 1).

Brain regions investigated were frontal cortex (BA8/9), temporal cortex (BA 21/22) to include the entorhinal cortex and hippocampus (at the level of the geniculate bodies), occipital cortex (BA17/18), cerebellar cortex, and brainstem to include substantia nigra (SN), LC and DRN (where available) and corpus striatum (CS) (caudate nucleus and putamen). Adjacent sections were routinely stained by haematoxylin and eosin, and immunostained for tau (using mouse monoclonal antibody, AT8 (Innogenetics, Antwerp, Belgium, 1:750)) and $A \beta$ (using mouse monoclonal antibody, 4G8 (Covance Research Products Inc., Dedham MA, USA, 1:3000). Following 4G8 and AT8 immunostaining, all cases were staged as to Thal phase of $\mathrm{A} \beta$ deposition [46] and Braak stage of neurofibrillary degeneration using the revised Braak criteria for AT8 immunostained paraffin sections [7]. Further sets of sections of brainstem (substantia nigra $(n=27)$ and/or locus caeruleu $(n=56)$ ) and temporal lobe with entorhinal cortex and hippocampus were immunostained for phosphorylated $\alpha$-synuclein using a polyclonal anti-psyn 1175 antibody [36] at 1:1000 dilution (kind gift of Dr. Masato Hasegawa at Tokyo Metropolitan Institute of Medical Science, Japan), and an additional set of sections of temporal cortex with hippocampus was immunostained for phosphorylated and non-phosphorylated TDP-43 using a polyclonal antibody (10782-2-AP Proteintech, Manchester, 1:1000). Those sections immunostained for tau, $\alpha$-synuclein and TDP-43 underwent antigen retrieval by pressure cooking in citrate buffer for $30 \mathrm{~min}$, reaching 120 degrees Celsius and $>15 \mathrm{kPa}$ pressure whereas sections immunostained for $A \beta$ were subject to incubation in $90 \%$ formic acid for $5 \mathrm{~min}$ as pretreatment.

Sections were also examined microscopically for the appearance, severity and topographical distribution of immunostaining of $A \beta$ within brain parenchyma (as amyloid plaques) and cerebral vessels (as CAA). A five-point 
Table 1 Selected demographic, genetic and neuropathological details for 56 individuals with Down syndrome

\begin{tabular}{|c|c|c|c|c|c|c|c|c|c|}
\hline Case ID\# & UKBBN ID\# & gender & age & karyotype & $A P O E$ & Thal & Braak & Allen CAA & Thal CAA \\
\hline 1 & na & $M$ & 0.01 & na & na & 0 & 0 & 0 & 0 \\
\hline 2 & na & $\mathrm{F}$ & 0.1 & na & na & 0 & 0 & 0 & 0 \\
\hline 3 & na & M & 1.6 & na & na & 0 & 0 & 0 & 0 \\
\hline 4 & na & M & 1.6 & na & na & 0 & 0 & 0 & 0 \\
\hline 5 & na & $\mathrm{F}$ & 3 & na & na & 0 & 0 & 0 & 0 \\
\hline 6 & na & M & 11 & na & na & 0 & 0 & 0 & 0 \\
\hline 7 & na & $\mathrm{F}$ & 13 & na & na & 0 & 0 & 0 & 0 \\
\hline 8 & BBN_2966 & M & 13 & $47 X Y 21$ & 3,3 & 1 & 0 & 0 & 0 \\
\hline 9 & na & M & 23 & na & na & 0 & 0 & 0 & 0 \\
\hline 10 & na & $\mathrm{F}$ & 27 & na & na & 1 & 0 & 0 & 0 \\
\hline 11 & na & M & 35 & na & na & 2 & 0 & 0 & 0 \\
\hline 12 & na & M & 36 & na & na & 5 & $\|$ & 0 & 0 \\
\hline 13 & BBN_2963 & $\mathrm{F}$ & 37 & $47 X X 21$ & 3,4 & 5 & I & 0 & 0 \\
\hline 14 & na & F & 39 & na & na & 2 & $b^{*}$ & 0 & 0 \\
\hline 15 & na & M & 42 & na & na & 5 & V & 1 & 1 \\
\hline 16 & BBN_17186 & $\mathrm{F}$ & 47 & na & na & 5 & $\mathrm{Vl}$ & 3 & 2 \\
\hline 17 & BBN_17189 & M & 47 & na & na & 5 & III & 1 & 1 \\
\hline 18 & BBN_17178 & $\mathrm{F}$ & 49 & na & 3,4 & 4 & III & 1 & 1 \\
\hline 19 & BBN_2981 & M & 50 & $47 X Y 21$ & 3,4 & 3 & $\|$ & 1 & 1 \\
\hline 20 & na & $\mathrm{F}$ & 50 & na & na & 0 & $b^{*}$ & 0 & 0 \\
\hline 21 & na & M & 51 & na & na & 5 & $\mathrm{Vl}$ & 1 & 1 \\
\hline 22 & BBN_2968 & M & 53 & $47 X Y 21$ & 3,3 & 5 & III & 1 & 1 \\
\hline 23 & BBN_3356 & $\mathrm{F}$ & 53 & na & 3,4 & 5 & $\mathrm{Vl}$ & 2 & 1 \\
\hline 24 & BBN_16954 & M & 54 & na & 2,4 & 5 & $\mathrm{Vl}$ & 3 & 2 \\
\hline 25 & BBN_3365 & M & 55 & na & 3,4 & 5 & $\mathrm{Vl}$ & 2 & 1 \\
\hline 26 & na & M & 55 & na & na & 5 & V & 1 & 1 \\
\hline 27 & BBN_3438 & F & 56 & na & 3,3 & 5 & $\mathrm{Vl}$ & 1 & 1 \\
\hline 28 & BBN_16273 & F & 56 & na & 3,4 & 5 & $\mathrm{Vl}$ & 1 & 1 \\
\hline 29 & BBN_2978 & M & 57 & $47 X Y 21$ & 3,3 & 5 & V & 3 & 2 \\
\hline 30 & na & M & 57 & na & na & 5 & $\mathrm{Vl}$ & 1 & 1 \\
\hline 31 & BBN_3020 & $\mathrm{F}$ & 58 & $47 X X 21$ & 3,3 & 5 & V & 1 & 1 \\
\hline 32 & BBN_3355 & M & 58 & na & 3,4 & 5 & $\mathrm{Vl}$ & 3 & 2 \\
\hline 33 & BBN_3358 & $\mathrm{F}$ & 58 & na & 3,3 & 5 & $\mathrm{Vl}$ & 2 & 1 \\
\hline 34 & BBN_3437 & $\mathrm{F}$ & 58 & na & 2,3 & 5 & V & 1 & 1 \\
\hline 35 & BBN_3353 & M & 59 & na & 2,3 & 5 & $\mathrm{Vl}$ & 3 & 2 \\
\hline 36 & BBN_3363 & M & 59 & na & 3,3 & 5 & $\mathrm{Vl}$ & 2 & 1 \\
\hline 37 & BBN_2990 & M & 60 & $47 X Y 21$ & 3,3 & 5 & $\mathrm{Vl}$ & 2 & 1 \\
\hline 38 & BBN_3364 & M & 60 & na & 3,3 & 5 & $\mathrm{Vl}$ & 2 & 1 \\
\hline 39 & na & $\mathrm{F}$ & 60 & na & na & 5 & 0 & 0 & 0 \\
\hline 40 & na & M & 60 & na & na & 5 & $\mathrm{Vl}$ & 2 & 1 \\
\hline 41 & BBN_16835 & M & 60 & na & 3,3 & 5 & III & 2 & 1 \\
\hline 42 & BBN_3352 & $\mathrm{F}$ & 61 & na & 2,3 & 5 & $\mathrm{Vl}$ & 3 & 2 \\
\hline 43 & BBN_3439 & $\mathrm{F}$ & 61 & na & 3,4 & 5 & $\mathrm{Vl}$ & 2 & 1 \\
\hline 44 & BBN_2964 & $\mathrm{F}$ & 62 & $47 X X 21$ & 3,3 & 5 & V & 3 & 2 \\
\hline
\end{tabular}


Table 1 Selected demographic, genetic and neuropathological details for 56 individuals with Down syndrome (Continued)

\begin{tabular}{|c|c|c|c|c|c|c|c|c|c|}
\hline Case ID\# & UKBBN ID\# & gender & age & karyotype & APOE & Thal & Braak & Allen CAA & Thal CAA \\
\hline 45 & BBN_2965 & $M$ & 62 & $47 X Y 21$ & 3,3 & 5 & V & 1 & 1 \\
\hline 46 & BBN_2967 & $\mathrm{F}$ & 62 & $47 X \times 21$ & 3,3 & 5 & IV & 3 & 2 \\
\hline 47 & BBN_3354 & M & 62 & na & 3,3 & 5 & $\mathrm{Vl}$ & 3 & 2 \\
\hline 48 & BBN_3441 & M & 62 & na & 2,3 & 5 & $\mathrm{Vl}$ & 3 & 2 \\
\hline 49 & na & M & 63 & na & na & 5 & $\mathrm{Vl}$ & 1 & 1 \\
\hline 50 & BBN_2969 & M & 64 & $47 X Y 21$ & 3,3 & 5 & IV & 1 & 1 \\
\hline 51 & BBN_3440 & $\mathrm{F}$ & 64 & na & 3,3 & 5 & $\mathrm{Vl}$ & 2 & 1 \\
\hline 52 & BBN_2975 & M & 65 & $47 X Y 21$ & 3,3 & 5 & V & 1 & 1 \\
\hline 53 & BBN_17004 & M & 65 & na & 3,3 & 5 & V & 1 & 1 \\
\hline 54 & na & M & 66 & na & na & 5 & V & 1 & 1 \\
\hline 55 & na & $\mathrm{F}$ & 69 & na & na & 5 & $\mathrm{Vl}$ & 1 & 1 \\
\hline 56 & BBN_2985 & M & 76 & $47 X Y 21$ & 2,3 & 5 & III & 3 & 2 \\
\hline
\end{tabular}

Thal = Thal phase of amyloid deposition and Braak = Braak tau stage. CAA phenotype was assessed in accordance with Allen et al. (2014) and Thal et al. (2010). UKBBN = UK Brain Bank Network ID number, na = not available/applicable

scoring system [31, 37] was employed to separately grade the severity of plaques and CAA.

\section{Plaques}

Grade 0 - no amyloid plaques in present.

Grade 1 - A few amyloid plaques in each low power ( $\times 10$ microscope objective) field.

Grade 2 - A moderate number of amyloid plaques in each low power field.

Grade 3 - Many dispersed amyloid plaques in each low power field.

Grade 4 - Very many, densely packed amyloid plaques in each low power field.

\section{CAA}

Grade 0 - No CAA in blood vessel walls in leptomeninges or brain parenchyma.

Grade 1 - Occasional blood vessels with CAA in leptomeninges and/or brain parenchyma, usually not occupying the full thickness of the wall. Grade 2 - A moderate number of blood vessels with CAA in leptomeninges and/or brain parenchyma, some occupying the full thickness of the wall. Grade 3 - Many blood vessels with CAA in leptomeninges and/or brain parenchyma, some occupying the full thickness of the wall.

Grade 4 - Most or all blood vessels with severe CAA in leptomeninges and/or brain parenchyma, occupying the full thickness of the wall.

CAA subtype, based on examination of frontal, temporal and occipital cortex in sections immunostained for $A \beta$, was assigned to all cases. Two classification systems were employed, as previously described [2, 47]. According to Allen et al. [2], Type 1 describes cases predominantly with many diffuse and cored $\mathrm{A} \beta$ plaques, throughout the cerebral cortex, in which CAA is confined within leptomeningeal vessels. Type 2 describes cases where, along with many diffuse and cored $A \beta$ plaques, CAA is present in both leptomeningeal and deeper penetrating arteries, especially within occipital cortex. Type 3 describes cases where capillary CAA is present along with arterial CAA, especially within primary visual cortex, but with relatively few $A \beta$ plaques. Type 4 describes a predominantly vascular phenotype, where $A \beta$ deposition is much more prevalent in and around blood vessels throughout the brain and A $\beta$ plaques are scarce or absent. According to Thal et al. [47] type 1 CAA includes both leptomeningeal and parenchymal vascular involvement and type 2 additionally involves capillaries.

The severity of overall tau (AT8) pathology (which included neurofibrillary tangles, neuritic plaques and neuropil threads) was assessed as:

Grade 0 - no tau pathology present.

Grade 1 - few, widely scattered neurofibrillary

tangles and/or neuropil threads in each low power

( $\times 10$ microscope objective) field.

Grade 2 - a moderate number of clustered neurofibrillary tangles and/or neuropil threads in each low power field.

Grade 3 - many clustered neurofibrillary tangles and/or neuropil threads in each low power field.

Grade 4 - many densely packed neurofibrillary tangles and/or neuropil threads in each low power field.

A similar system was employed to score $\alpha$-synuclein immunostained Lewy body and Lewy neurite pathology 
in $\mathrm{SN}$ and $\mathrm{LC}$, entorhinal and temporal cortex, and for TDP-43 immunopositive neuronal cytoplasmic inclusions in the dentate gyrus of the hippocampus and fusiform gyrus of the temporal lobe.

\section{APOE genotyping}

DNA was extracted from frozen cerebellum (or frontal cortex when cerebellum was not available) by routine methods; frozen tissues were not available for genotyping in 24 cases (Table 1). APOE genotyping was performed on all samples using method of Wenham et al. [49].

\section{Statistical analysis}

All data analysis was performed using SPSS $\mathrm{v}$ 21.0. Levels of significance were two-tailed and set at $p<0.05$.

\section{Results}

\section{Histological changes}

Amyloid pathology

Of the 56 individuals, 8 (cases \#1-7 and 9) showed no amyloid plaques whatsoever in any brain region examined. Five of these (cases \#1-5) were aged 3 years or under, two (cases \#6 and 7) were aged 11 and 13 years, and one (case \#9) was 23 years of age. Of the remaining 48 individuals, three, aged 13, 27, and 50 years (cases \#8, 10 and 20, respectively), showed only rare to moderate numbers of diffuse amyloid plaques (Fig. 1a). In the 27 and 50-year olds these occurred within the temporal cortex alone, but were also present in frontal and occipital cortex in the 13-year old. None were present in the hippocampus, corpus striatum, cerebellum or brainstem of any of these 3 individuals. The other 45 individuals all showed either a moderate number of, or many, amyloid plaques (Fig. 2). However, in 5 of these (cases \#11-14 and 19, aged 35, 36, 37,39 and 50 years, respectively) plaques were mostly diffuse with only few cored, neuritic amyloid plaques being seen within the temporal neocortex and hippocampus, whereas in all other individuals over 50 years of age plaque density and morphology was similar to that typically seen in AD. Hence, cases \#1-7 and \#9 (where no amyloid plaques were present) corresponded to Thal phase 0 , cases \#8, 10 and 20 to Thal phase 1 , cases \#11 and 14 to Thal phase 2 , case \#19 to Thal phase 3, case \#18 to Thal phase 4 with all other cases corresponding to Thal phase 5 (Fig. 2).

\section{Cerebral amyloid angiopathy}

Fifteen (of twenty) individuals aged 50 years or below showed no CAA at all. CAA was seen only in 5 individuals (cases \#15-19) (Fig. 2). In case \#15 (aged 42 years) CAA sparsely affected leptomeningeal arteries in the temporal and occipital cortex, but CAA was also present in a few leptomeningeal arteries of the frontal, temporal and occipital cortex and cerebellum in case \#19. In the other 3
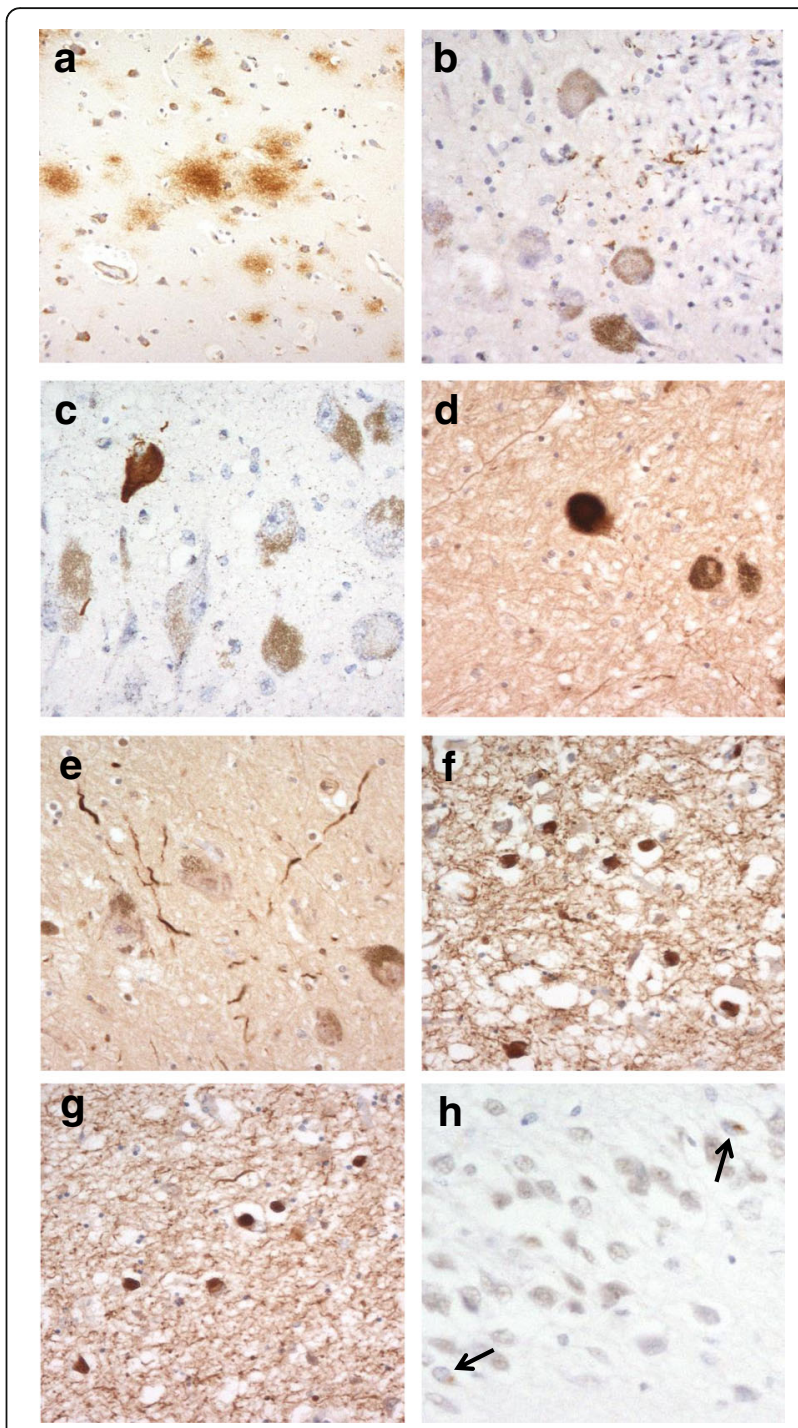

Fig. 1 Pathological changes in Down syndrome. In case \#8, aged 13 years, amyloid deposits are present in the temporal cortex as diffuse plaques in the absence of any tau pathology (a). A few tau positive neurites are present in locus caeruleus in case \#14 (b) and a single tau positive neurofibrillary tangle is seen, again in locus caeruleus, in case \#20 (c), in the absence of any tau pathology elsewhere in the brain. A few a-synuclein positive Lewy bodies (d) and Lewy neurites (e) are present in the substantia nigra in case \#45, aged 62 years, but these are more densely present in entorhinal cortex (f) and temporal neocortex ( $\mathbf{g}$ ) of the same case. Sparse TDP-43 neuronal cytoplasmic inclusions (arrowed) are seen in dentate gyrus granule cells in case \#43, aged 61 years (h). Immunoperoxidase-haematoxylin; $\times 250$ microscope magnification (a) $\times 400$ microscope magnification $(\mathbf{b}-\mathbf{h})$

individuals (cases \#16-18, aged 47, 47 and 49 years) CAA was moderate to severe and was present in leptomeningeal arteries in the frontal (except case \#16), temporal and occipital cortex and cerebellum (all 3 cases). Cases \#15, 18 and 19 therefore displayed type 1 CAA [2]. In case \#17 (aged 47 years) CAA also involved parenchymal arteries 


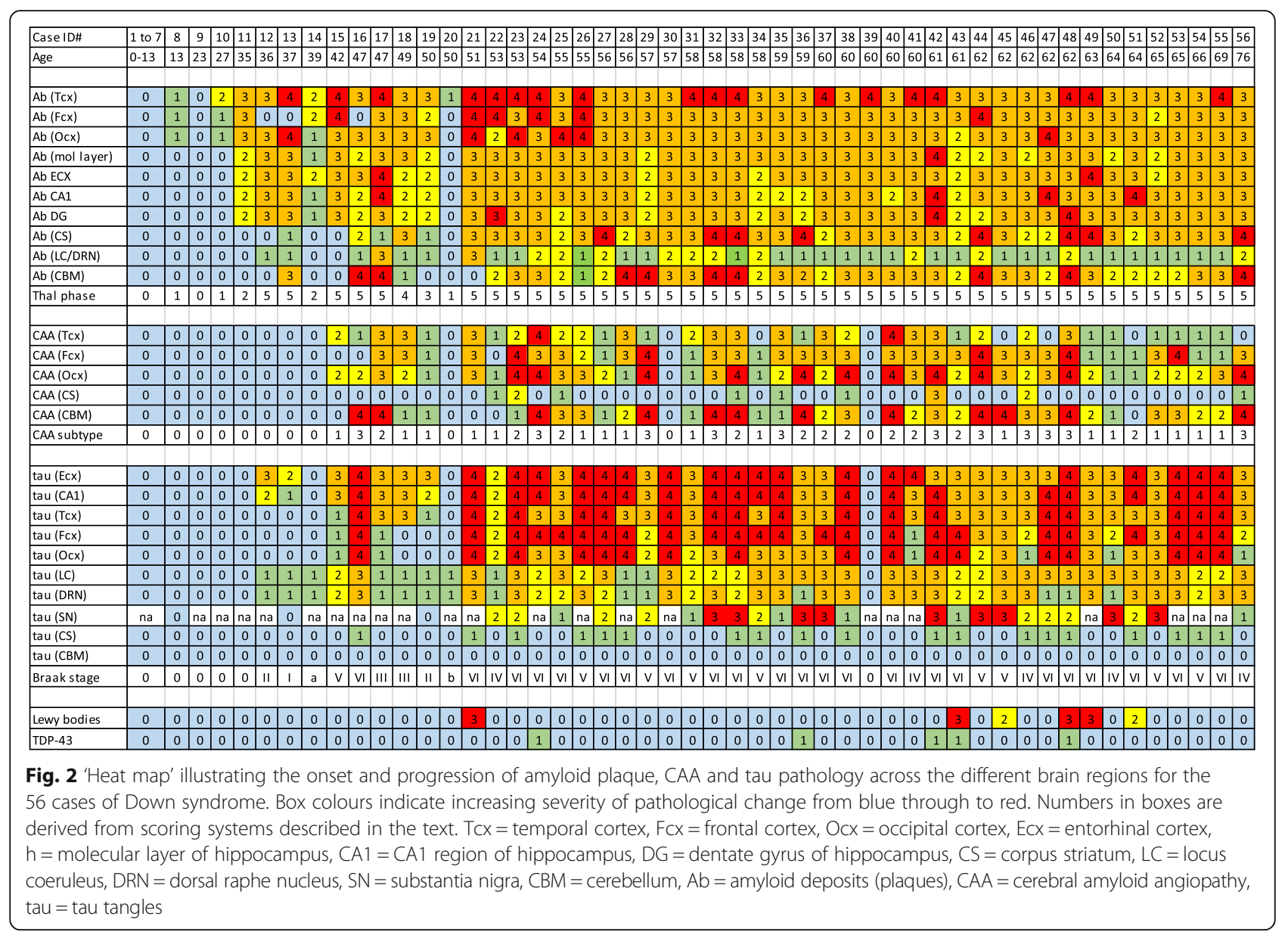

(type 2) and parenchymal arteries and capillaries (type 3 CAA) in case \#16.

All 36 individuals over 50 years of age (except cases \#30 and 39, aged 57 and 60 years) showed some degree of CAA (Fig. 2). In 14 individuals this was confined to leptomeningeal arteries (CAA type 1), being only mild to moderate in 10 of these, but severe or very severe in the other 6 . In 10 individuals, CAA involved intraparenchymal arteries as well as leptomeningeal arteries (CAA type 2), at least in occipital cortex, often also in frontal and/or temporal cortex, but not in cerebellum. In the remaining 10 individuals there was capillary involvement as well as leptomeningeal and parenchymal artery involvement, again always in the occipital cortex, but occasionally also in the frontal cortex.

Overall, therefore, CAA was present in 39 individuals. According to Allen et a criterial [2] this was present as type 1 CAA in 17 of these (44\%), type 2 in 11 individuals (28\%) and type 3 in 11 individuals (28\%) (Table 1, Fig. 2). According to Thal et al. criteria [47], CAA was present as type 1 in $72 \%$ individuals and type 2 in $28 \%$ individuals (Table 1).

Mild CAA was seen in small arteries in CS in only 9 individuals (Fig. 2).

\section{Tau pathology}

Of the 56 individuals, 14 showed no tau tangles or neuropil threads whatsoever in entorhinal cortex, hippocampus or neocortex. Eleven of these (cases \#1-11) were aged 35 years or under, one (case \#14) was aged 39 years, one (case \#20) was 50 years of age and one (case \#39 was 60 years of age. Interestingly, in cases \#14 and \#20, there was scant tau neuritic (Fig. 1b) or neurofibrillary (Fig. 1c) pathology in LC, but without any involvement of cortical or other subcortical structures. Such cases might be classed as pretangle/prodromal stage 'a' or 'b', respectively (see $[6,8]$ - a stage recently postulated to predate Stage I in earlier stageing systems [4, 7]. In case \#39 no tau pathology at all was seen in any brain region.

Of the other 42 individuals, 10 (cases \# 12, 13, 17-19, 22, 41, 46, 50 and 56 aged 36, 37, 47, 47, 50, 53, 60, 62, 64 and 76 years, respectively) showed only a moderate number of, or many tangles within the hippocampus (and entorhinal cortex), with only rare, or a moderate number of, tangles in the temporal, frontal or occipital cortex. These were considered to be at Braak stages II-IV. The remaining 32 individuals (30 of whom were over 50 years of age) showed a moderate number of, many, or very many, tangles in all 
neocortical regions and hippocampus, similar in appearance, distribution and degree to that typically seen in $\mathrm{AD}$, and were assessed as being at Braak stages V or VI (Fig. 2).

Tau pathology was also investigated in $\mathrm{SN}$ in 27 cases where this region was available. No tau pathology was present in any case under 50 years of age, but rapidly developed thereafter such that this was present as neurofibrillary tangles and neuropil threads in all cases examined who were older than 50 years of age, ranging from moderate numbers of both through to there being very many present (Fig. 2).

No tau pathologies consistent with Ageing Related Tau Astrogliopathy (ARTAG) [22] or Argyrophilic Grain Disease [5] were seen in any of the studied cases.

\section{a-Synuclein pathology}

a few to a moderate number of $\alpha$-synuclein immunopositive Lewy bodies (Fig. 1d) and Lewy neurites (Fig. 1e) were present in SN and/or LC in 5 cases (\#21, 43, 45, 48 and 49), all over 50 years of age, but both pathologies were numerous in the entorhinal cortex (Fig. 1f) and moderately present in the temporal cortex (Fig. 1g), in the same 5 cases and also in case \#51 where none were present in the SN or LC. Loss of neurones from SN was generally absent or sparse, even in those cases where Lewy body pathology was present.

\section{TDP-43 pathology}

TDP-43 pathology was absent from hippocampus and fusiform gyrus/temporal cortex in all but 5 cases (cases \#24, 36, 42, 43 and 48, aged 54, 59, 61, 61 and 62 years, respectively). In these latter 5 cases sparse granular neuronal cytoplasmic inclusions were present in cells of the dentate gyrus of the hippocampus (Fig. 1h), but none were seen in fusiform gyrus or temporal cortex. In 3 cases (cases \#42, 43 and 47) hippocampal sclerosis of CA1 was also present.

\section{Other pathologies}

We carefully examined routine haematoxylin eosin stained sections for evidence of small vessel disease, microinfarctions, overt microbleeds or intracerebral haemorrhage but found none to be consistently present in any patient or CAA subtype.

\section{Early sites of pathological changes}

Figure 2 shows a 'heat map' illustrating the earliest sites, and progression, of amyloid plaque, CAA and tau pathology across the different brain regions for the 56 cases.

Amyloid pathology commences in late teens to late twenties as a deposition of diffuse plaques initially within temporal lobe (Thal phase 1), quickly involving other neocortical regions and hippocampus (Thal phase 2), but only reaching subcortical regions (Thal phases 3 and
4) and cerebellum (Thal phase 5) by the late forties. After 50 years of age, every region of the brain, including cerebellum and corpus striatum, is moderately or heavily invested with amyloid plaques (Thal phase 5). Interestingly, CAA does not regularly commence until after 4550 years of age. In terms of tau pathology and Braak stageing, there is a progressive 'spread' of neurofibrillary pathology throughout the brain, usually commencing after the age of 35 years. This initially involves not only entorhinal areas and hippocampus but also subcortical regions such as LC and DRN, sometimes in the absence of entorhinal involvement. Later, tau pathology spreads throughout the neocortex reaching occipital lobes (Braak stages $\mathrm{V}$ or $\mathrm{VI}$ ) in most instances by mid-50 years of age. Lewy body pathology and TDP-43 pathological changes were infrequently present and generally only seen in individuals over $50-60$ years of age.

\section{APOE genotyping}

APOE genotypes are given in Table 1 . There were 5 individuals bearing $\varepsilon 2 \varepsilon 3$ genotype, 18 bearing $\varepsilon 3 \varepsilon 3$ genotype, 8 bearing $\varepsilon 3 \varepsilon 4$ genotype and 1 bearing $\varepsilon 2 \varepsilon 4$ genotype. There were no $\varepsilon 2$ or $\varepsilon 4$ homozygotes. These genotypes provided $A P O E \varepsilon 2$ and $\varepsilon 4$ allele frequencies of 7.8 and $14.0 \%$, respectively. However, age at death was earlier in those individuals bearing one APOE \&4 allele (52.6 \pm 6.9 years) compared to those bearing only $A P O E \varepsilon 3$ alleles $(57.8 \pm 11.6$ years, $p=0.158)$ and those with $A P O E$ $\varepsilon 2$ alleles $(63.2 \pm 7.3$ years, $p=0.019)$, the latter, in turn, being later than in those individuals bearing only $A P O E$ $\varepsilon 3$ alleles $(p=0.232)$. There were no associations between $A P O E \varepsilon 2$ or $\varepsilon 4$ alleles and the severity of $\mathrm{A} \beta$ plaque deposition (Thal phase), CAA subtype or severity of tau pathology (Braak stage).

\section{Discussion}

Although post mortem studies of established cases of AD have identified the major cell types and functional systems affected by the disorder, the initial sites of pathology are still unclear. According to the 2006 Braak stageing protocol [7], the earliest sites of tau pathology lie within the entorhinal and transentorhinal cortex (stage I), spreading to hippocampus (stage II), temporal cortex (stage III) and eventually to other regions of cerebral cortex (stage IV), finally reaching visual association cortex (stage $\mathrm{V}$ ) and primary visual cortex (stage VI). However, this classification has recently been modified and stages prior to the entorhinal involvement affecting subcortical nuclei such as LC and DRN (pre-tangle/prodromalstages a-c) in isolation from any cortical involvement have been proposed $[6,8]$. Conversely, the order of amyloid plaque deposition [46] commences within neocortical regions, particularly within the temporal lobe (phase 1), and spreads to involve allocortical regions such as the hippocampus and amygdala 
(phase 2), then into subcortical regions (phase 3), brain stem (phase 4) and finally cerebellum (phase 5). However, even though both of these protocols were based on a reasonably large number of demented and non-demented individuals (83 individuals were included in the original Braak tau stageing in 1991, and again in 2006 in the modified criteria, with 47 being employed for Thal phase protocols) the data generated still represents an assimilation of single time point, cross sectional observations into a continuum of change. The stageing protocols are therefore based on the presumption that 'normal' cases with limited, and presumed early, non-clinical pathological changes would have progressed into ones with dementia and fully developed pathology had they lived longer, and conversely those with full blown AD pathology would have flowed through the same, more limited, stages earlier in the development of disease. While this is a reasonable presumption, it still remains as such given the fact that the data are generated from a heterogeneous collection of heathy and demented individuals where the same risk factors for disease onset and progression may not necessarily apply. The advantage of studying individuals with DS lies mainly in the fact that they are a more homogenous study group who largely share the same genetic (and to some extent environmental) risk of disease, and are therefore a more predictable 'model' of disease onset and progression than are (selected) members of the general population.

Consequently, when comparing the origin and spread of tau and amyloid pathology (see Fig. 2) in this series of individuals with DS, it is clear that amyloid pathology occurred in the absence of tau pathology in 3 of 4 individuals aged between 13 and 35 years; neither were present in one 23-year old. Initially, this was present broadly to a similar extent in all regions of cerebral cortex, progressing with time into hippocampus, striatum and cerebellum. A pattern typical of AD [46] was reached by $50-55$ years of age. These findings bear out our previous studies on more limited numbers of cases $[28,29]$ and are consistent with those of other workers where cases showing amyloid deposits in the absence of tau can be seen in individuals as young as 8 years of age $[23,24]$. Early deposits of amyloid $(A \beta)$ are seen as diffuse plaques and to be composed of the longer form $A \beta_{42(3)}$, with the shorter form, $A \beta_{40}$, only appearing later in (more advanced) cored plaques [20]. It has been suggested that deposition of amyloid in very young individuals with DS may have occurred in relationship to an institutionalized residential background [23]. In these present study, a mix of institutionalised and community based individuals were studied though no clear difference in pattern of amyloid deposition was seen between such individuals where this information was definitely available.
Interestingly, in the present study, amyloid deposition in blood vessel walls (CAA) did not commence before 42 years of age, some 30 years after initial deposition of $\mathrm{A} \beta$ as plaques. Similar findings have recently been reported by Head et al. [15]. It has been postulated that the strong association between age, CAA and AD pathology in the general population is driven, at least partially, by an impaired efficiency of cerebral vessels in later life in expelling extracellular fluid containing soluble forms of $A \beta$ as a consequence of atherosclerosis/arteriosclerosis within such vessels [48]. However, individuals with DS appear to be protected against hypertension [1] and show less cerebrovascular pathology typically associated with cardiovascular risk factors, including atherosclerotic lesions and arteriolosclerosis $[15,27]$. Paradoxically, this ought to result in a better preservation of perivascular drainage channels in DS, and consequently less severe CAA compared to aged persons in the general population with AD. Potential inefficiencies in perivascular drainage might only appertain to those few very elderly individuals with DS who might survive to an age bordering on those in the general population. However, $A \beta$ can also be cleared from the brain through several other routes, involving endocytosis by microglia and astrocytes, or enzymatic degradation. It is therefore possible that failures in these latter pathways in younger persons with DS might foster an inability to expel $A \beta$ and result in CAA.

As previously reported $[18,43,44], A P O E$ alleles and genotypes were similar to those in the general population, and in contrast to $\mathrm{AD}$, there was no elevation of $A P O E \& 4$ allele (or reduction in $\varepsilon 2$ allele) frequency. Nonetheless, again as reported previously [18, 41, 43, 51], age at death was earlier in $\varepsilon 4$ allele bearers than in those without $\varepsilon 4$ allele and was later in $\varepsilon 2$ allele bearers than in those without $\varepsilon 2$ allele suggesting that possession of $A P O E$ $\varepsilon 4$ allele may hasten the onset of dementia and mortality by promoting an earlier formation, or a more rapid accumulation, of plaques and tangles. Although possession of $A P O E \varepsilon 4$ allele, and particularly homozygosity for this, has been reported to favour CAA at capillary level in persons with $\mathrm{AD}[37,45,47]$ we found no association in DS between $A P O E$ \&4 allele and type 3 CAA [2] (or type 2 CAA as in [47]).

On the other hand, tau pathology did not commence anywhere in the brain until after 35 years of age, though its onset in most cases appeared synchronous within both entorhinal/transentorhinal areas and subcortical nuclei (LC and DRN), with hippocampus and neocortical regions only becoming involved after $45-50$ years of age. However, in two instances (cases \#14 and 20) there was scant neuritic/neurofibrillary pathology in LC without any involvement of other cortical or subcortical structures. Such cases might be classed as 'prodromal stages 'a' or ' $b$ ' $[6,8]$. Nonetheless, thereafter pattern of spread of tau 
pathology in DS is similar to that seen in typical AD [4, 7]. Hence, observations in DS strongly validate present stageing protocols for tau and amyloid pathology, and provide the clearest 'model' yet for investigation the origins and progress of pathological changes typical of AD.

While most cases studied fitted in well with this age-increase pattern of spread of amyloid and tau pathology, though there were 2 notable exceptions, cases \#20 and \#39, which did not conform to pathological expectations given their chronological age. In case \#20, aged 50 years, virtually no amyloid, and no tau pathology, was present, while in case \#39 there was abundant amyloid pathology, though tau pathology was absent. It is possible that these individuals bore a karyotype other than full trisomy 21 since it is known that cases bearing only partial trisomy excluding the APP locus, but covering the obligate DS region, show little or no Alzheimer type pathology, even at advanced age [12, 40]. Unfortunately, a karyotype was not available for these 2 cases.

The low prevalence (9\% cases) of TDP-43 pathological changes in hippocampus and fusiform gyrus is consistent with previous studies and parallels that seen in cases of sporadic and inherited early onset AD [11,26]. This type of TDP-43 proteinopathy is much more common in late onset cases of AD [11,34] at a time beyond the lifespan of people with DS. The higher prevalence of TDP-43 pathology in late onset $\mathrm{AD}$ compared to early onset $\mathrm{AD}$ and DS may be triggered as part of an age-associated process operating in conjunction with $\mathrm{AD}$ at a later time of life. Nonetheless, it is also possible that the presence of $\mathrm{AD}$ pathology per se initiates and promotes TDP-43 pathology. However, if this were so then a higher prevalence of TDP-43 pathology might be expected in DS than that which is seen given the severity of AD pathology occurring in older persons with DS.

A previous study has reported that $\alpha$-synuclein immunopositive Lewy bodies and Lewy neurites are common in older individuals with DS, especially within the amygdaloid nucleus where as many as half of cases can show such changes [25]. In the present report, Lewy bodies and Lewy neurites were present in SN or LC or both in only 5 cases (9\%) and were generally few or moderate in number. In these cases, no clear loss of nigral neurones had occurred. On the other hand, Lewy bodies and Lewy neurites were much more common in the entorhinal cortex and, to a lesser extent, temporal cortex, but again occurred only in $6(11 \%)$ cases (which included the 5 cases where SN or LC was affected). Unfortunately, the amygdaloid nucleus was not available for investigation in the present study, though given the absence of Lewy body pathology in entorhinal cortex in most cases it is unlikely that these negative cases would have shown (at least florid) changes in the amygdala. The reason for the apparent lower frequency of Lewy body pathology in the present series of cases compared to that reported by Lippa and colleagues [25] is not clear given that both series examined individuals of a similar age range and employed $\alpha$-synuclein immunostaining, albeit with different antibodies, though may be explained by the lack of investigation of the amygdala in our own study.

We did obtain some evidence (from cases \#14 and 20) supporting the view $[6,11]$ that tau pathology in subcortical nuclei might predate that within entorhinal/transentorhinal areas, and that there might be stages in the tau pathological process before these latter cerebral cortical regions become affected. On the other hand, we observed that in 3 of the youngest individuals amyloid pathology preceded that of tau, and conversely that tau pathology was never seen in the absence of $A \beta$ deposition - findings again consistent with the amyloid cascade hypothesis of AD [14]. Such findings accord with the view that pathological changes in tau might in some way be induced by damage to nerve terminals at the sites of amyloid plaque formation, with retrograde responses in the perikaryon leading to NFT formation. In the case of LC and DRN neurons this would occur at plaque sites within the cerebral cortex (and particularly the temporal cortex), since it is known that these nerve cell types project diffusely, but topographically, all cortical regions. Indeed, in DS and AD nerve cells of LC which project to cortical regions rich in plaques are lost whereas those projecting to relatively plaque free regions such as cerebellum, and plaque free spinal cord, are preserved [32, 33]. In entorhinal/transentorhinal areas induction of damage would take place at plaque sites within the molecular layer of the hippocampus where such neurons project via the perforant pathway [17]. The observations that tau pathology only became prevalent in SN after the age of 50 years - some 1015 years after LC and DRN - would accord with the later deposition (relative to the cerebral cortex) of $A \beta$ within the caudate nucleus and putamen, regions where these neurons make their principal connections.

On the other hand, it is possible that tau aggregation precedes and induces amyloid deposition in some way. Indeed, previous work based on cognitively normal young individuals suggests that areas such as LC might be the initial site of tau pathology in $\mathrm{AD}[6,8]$. However, the absence of amyloid deposits in the brains of such normal individuals implies a pathological process at work other than one that leads to $\mathrm{AD}$, unless it is accepted that tau pathology in some way induces amyloid pathology at a later stage in persons other than those with DS. It is also possible that the tau pathology seen in these very young individuals is indicative of primary age related tauopathy (PART), where tau pathology occurs, especially in entorhinal/transentorhinal regions, in the relative or complete absence of amyloid deposition [10]. However, PART is generally associated with aged individuals, and an onset of this tau pathology in youth with maintenance through 
into old age seems unlikely. Tau pathological changes in LC and entorhinal cortex, in the absence of significant amyloid deposits, can also be seen in many patients with other neurodegenerative disorders such as Amyotrophic Lateral Sclerosis [3] or Huntington's disease [21]. Furthermore, it is known that a reversible PHF-like pattern of tau pathology can be induced under certain physiological conditions such as hypothermia and anaesthesia [39]. Therefore, it remains possible that neurons of LC (as well as others such as those in DRN and nucleus of Meynert) are exquisitely vulnerable to a range of physiological or neurotoxic changes that induce tau pathology, and that these putative early changes in tau in LC in healthy young people in the absence of amyloid deposits $[6,8]$ might simply be serendipitous or even transient, never progressing into a pathological process culminating in AD.

Finally, it is possible that the initiation of tau and amyloid pathologies occur independently but once started each process might act synergistically to promote the progression of the other. It might even be the case that onset of tau pathology in persons with DS represents early onset of aging processes since it is well known that such individuals display other aspects of 'precocious aging' involving skin, hair or cardiac changes at an earlier time of life compared to people within the general population.

\section{Conclusion}

In conclusion, a study of the brains of individuals with DS at different ages reinforces the concepts underlying the amyloid cascade hypothesis of $\mathrm{AD}$ and supports present stageing protocols for the onset and spread of both amyloid and tau pathology.

\section{Acknowledgements \\ We acknowledge the support of the Manchester Brain Bank by Alzheimer's Research UK and Alzheimer's Society through their funding of the Brains for Dementia Research (BDR) Programme. Manchester Brain Bank also receives Service Support costs from Medical Research Council.}

\section{Funding}

Manchester Brain Bank receives funding from Alzheimer's Research UK and Alzheimer's Society, through the Brains for Dementia Research (BDR) Programme, and also receives Service Support Costs from Medical Research Council of UK.

\section{Authors' contributions \\ Study concept, design and manuscript preparation (DM, VP), microscopic assessments (DM), technical support and data analysis (AR, YD).}

\section{Ethics approval and consent to participate}

The study was approved by Manchester Brain Bank Management Committee (REC reference 09/H0906/52 + 5). Under conditions agreed with the Research Ethics Committee, The Manchester Brain Bank can supply tissue or data to researchers, without requirement for researchers to apply individually to the REC for approval.

\section{Consent for publication}

All authors have read and approved the final manuscript.

\section{Competing interests}

The authors declare that they have no competing interests.

\section{Publisher's Note}

Springer Nature remains neutral with regard to jurisdictional claims in published maps and institutional affiliations.

\section{Author details}

${ }^{1}$ Institute of Brain, Behaviour and Mental Health, Faculty of Medical and Human Sciences University of Manchester, Salford Royal Hospital, Stott Lane, Salford M6 8HD, England. '2South Birmingham Community NHS Trust, Birmingham, UK. ${ }^{3}$ Liverpool John Moores University, Liverpool, UK.

Received: 21 June 2018 Accepted: 21 June 2018

Published online: 04 July 2018

\section{References}

1. Alexander M, Petri H, Ding Y, Wandel C, Khwaja O, Foskett N (2016) Morbidity and medication in a large population of individuals with Down syndrome compared to the general population. Dev Med Child Neurol 58:246-254

2. Allen N, Robinson AC, Snowden S, Davidson YS, Mann DMA (2014) Patterns of cerebral amyloid angiopathy define histopathological phenotypes in Alzheimer's disease. Neuropathol Appl Neurobiol 40:136-148

3. Behrouzi R, Liu X, Wu D, Robinson AC, Tanaguchi-Watanabe S, Rollinson S et al (2016) Pathological tau deposition in motor Neurone disease and frontotemporal lobar degeneration associated with TDP-43 proteinopathy. Acta Neuropathol Commun 4:33

4. Braak H, Braak E (1991) Neuropathological stageing of Alzheimer-related changes. Acta Neuropathol 82:239-259

5. Braak H, Braak E (1998) Argyrophilic grain disease: frequency of occurrence in different age categories and neuropathological diagnostic criteria. J Neural Transm 105:801-819

6. Braak H, Del Tredici K (2011) The pathological process underlying Alzheimer's disease in individuals under thirty. Acta Neuropathol 12:171-181

7. Braak H, Alafuzoff I, Arzberger T, Kretzschmar H, Del Tredici K (2006) Staging of Alzheimer disease-associated neurofibrillary pathology using paraffin sections and immunocytochemistry. Acta Neuropathol 112:389-404

8. Braak H, Thal DR, Ghebremedhin E, Del Tredici K (2011) Stages of the pathologic process in Alzheimer disease: age categories from 1 to 100 years. J Neuropathol Exp Neurol 70:960-969

9. Burger PC, Vogel FS (1973) The development of the pathological changes of Alzheimer's disease and senile dementia in patients with Down's syndrome. Am J Pathol 73:457-476

10. Crary JF, Trojanowski JQ, Schneider JA, Abisambra JF, Abner EL, Alafuzoff I et al (2014) Primary age-related tauopathy (PART): a common pathology associated with human aging. Acta Neuropathol 128:755-766

11. Davidson YS, Raby S, Foulds PG, Robinson A, Thompson JC, Sikkink S et al (2011) TDP-43 pathological changes in early onset familial and sporadic Alzheimer's disease, late onset Alzheimer's disease and Down's syndrome: association with age, hippocampal sclerosis and clinical phenotype. Acta Neuropathol 122:703-713

12. Doran E, Keator D, Head E, Phelan MJ, Kim R, Totoiu M et al (2017) Down syndrome, partial trisomy 21, and absence of Alzheimer's disease: the role of APP. J Alzheimers Dis 56:459-470

13. Ellis WG, McCulloch JR, Corley CL (1974) Presenile dementia in Down's syndrome. Ultrastructural identity with Alzheimer's disease. Neurology 24:101-106

14. Hardy J, Allsop D (1991) Amyloid deposition as the central event in the aetiology of Alzheimer's disease. Trends Pharmacol Sci 12:383-388

15. Head E, Phelan MJ, Doran E, Kim RC, Poon WW, Schmidt FA et al (2017) Cerebrovascular pathology in Down syndrome and Alzheimer disease. Acta Neuropathol Comm 5:93

16. Hof PR, Bouras C, Perl DP, Sparks DL, Mehta N, Morrison JH (1995) Agerelated distribution of neuropathologic changes in the cerebral cortex of patients with Down's syndrome. Quantitative regional analysis and comparison with Alzheimer's disease. Arch Neurol 52:379-391

17. Hyman BT, Van Hoesen GW, Damasio AR, Barnes CL (1984) Alzheimer's disease: cell-specific pathology isolates the hippocampal formation. Science 225:1168-1170

18. Hyman BT, West HL, Rebeck GW, Lai F, Mann DM (1995) Neuropathological changes in Down's syndrome hippocampal formation. Effect of age and apolipoprotein E genotype. Arch Neurol 52:373-378 
19. Hyman BT, Phelps CH, Beach TG, Bigio EH, Cairns NJ, Carrillo MC et al (2012) National Institute on Aging-Alzheimer's association guidelines for the neuropathologic assessment of Alzheimer's disease. Alzheimers Dement 8:1-13

20. Iwatsubo T, Mann DMA, Odaka A, Suzuki N, Ihara Y (1995) Amyloid B-protein (Aß) deposition: $A \beta_{42(43)}$ precedes $A \beta_{(40)}$ in Down's syndrome. Ann Neurol 37:294-299

21. Jellinger KA (1998) Alzheimer-type lesions in Huntington's disease. J Neural Transm 105:787-799

22. Kovacs GG, Ferrer I, Grinberg LT, Alafuzoff I, Attems J, Budka H et al (2016) Aging-related tau astrogliopathy (ARTAG): harmonized evaluation strategy. Acta Neuropathol 131:87-102

23. Lemere CA, Blusztajn JK, Yamaguchi H, Wisniewski T, Saido TC, Selkoe DJ (1996) Sequence of deposition of heterogeneous amyloid beta-peptides and APOE in Down syndrome: implications for initial events in amyloid plaque formation. Neurobiol Dis 3:16-32

24. Leverenz JB, Raskind MA (1998) Early amyloid deposition in the medial temporal lobe of young Down syndrome patients: a regional quantitative analysis. Exp Neurol 150:296-304

25. Lippa CF, Schmidt ML, Lee VM, Trojanowski JQ (1999) Antibodies to alphasynuclein detect Lewy bodies in many Down's syndrome brains with Alzheimer's disease. Ann Neurol 45:353-357

26. Lippa CF, Rosso AL, Stutzbach LD, Neumann M, Lee VM, Trojanowski JQ (2009) Transactive response DNA-binding protein 43 burden in familia Alzheimer disease and Down syndrome. Arch Neurol 66:1483-1488

27. Lott IT, Head E (2005) Alzheimer disease and Down syndrome: factors in pathogenesis. Neurobiol Aging 26:383-389

28. Mann DMA (1988) The pathological association between Down syndrome and Alzheimer disease. Mech Ageing Dev 43:99-136

29. Mann DMA, Esiri MM (1988) The site of the earliest lesions of Alzheimer's disease. New Eng J Med 318:789-790

30. Mann DMA, Younis N, Jones D, Stoddart RW (1992) The time course of pathological events concerned with plaque formation in Down's syndrome with particular reference to the involvement of microglial cells. Neurodegeneration 1:201-215

31. Mann DMA, Davidson YS, Robinson AC, Allen N, Hashimoto T, Richardson A et al (2018) Patterns and severity of vascular amyloid in Alzheimer's disease associated with duplications and missense mutations in APP gene, Down syndrome and sporadic Alzheimer's disease. Acta Neuropathol. https://doi. org/10.1007/s00401-018-1866-3

32. Marcyniuk B, Mann DM, Yates PO (1986) The topography of cell loss from locus caeruleus in Alzheimer's disease. J Neurol Sci 76:335-345

33. Marcyniuk B, Mann DM, Yates PO, Ravindra CR (1988) Topography of nerve cell loss from the locus caeruleus in middle aged persons with Down's syndrome. J Neurol Sci 83:15-24

34. McAleese KE, Walker L, Erskine D, Thomas AJ, McKeith IG, Attems J (2017) TDP-43 pathology in Alzheimer's disease, dementia with Lewy bodies and ageing. Brain Pathol 20:472-479

35. Montine TJ, Phelps CH, Beach TG, Bigio EH, Cairns NJ, Dickson DW et al (2012) National Institute on Aging-Alzheimer's association guidelines for the neuropathologic assessment of Alzheimer's disease: a practical approach. Acta Neuropathol 123:1-11

36. Obi K, Akiyama H, Kondo $H$, Shimomura $Y$, Hasegawa M, Iwatsubo $T$ (2008) Relationship of phosphorylated a-synuclein and tau accumulation to $A \beta$ deposition in the cerebral cortex of dementia with Lewy bodies. Expl Neurol 210:409-420

37. Olichney JM, Hansen LA, Galasko D, Saitoh T, Hofstetter CR, Katzman R et al (1996) The apolipoprotein E epsilon 4 allele is associated with increased neuritic plaques and cerebral amyloid angiopathy in Alzheimer's disease and Lewy body variant. Neurology 47:190-196

38. Olson Ml, Shaw CM (1969) Presenile dementia and Alzheimer's disease in mongolism. Brain 92:147-156

39. Planel E, Richter KE, Nolan CE, Finley JE, Liu L, Wen Y et al (2007) Anaesthesia leads to tau hyperphosphorylation through inhibition of phosphatase activity by hypothermia. J Neurosci 27:3090-3097

40. Prasher VP, Farrer MJ, Kessling AM, Fisher EM, West RJ, Barber PC et al (1998) Molecular mapping of Alzheimer-type dementia in Down's syndrome. Ann Neurol 43:380-383

41. Prasher VP, Schupf N, Sajith SG, Zigman WB, Rees S, Patel A et al (2008) Significant effect of APOE epsilon 4 genotype on the risk of Alzheimer's disease and mortality in persons with Down syndrome. Int J Geriatr Psychiatry 23:1134-1140
42. Ropper AH, Williams RS (1980) Relationship between plaques and tangles and dementia in Down's syndrome. Neurology 30:639-644

43. Royston MC, Mann D, Pickering-Brown S, Owen F, Perry R, Raghavan R et al (1994) Apolipoprotein E epsilon 2 allele promotes longevity and protects patients with Down's syndrome from dementia. Neuroreport 5:2583-2585

44. Saunders AM, Schmader K, Breitner JC, Benson MD, Brown WT, Goldfarb L et al (1993) Apolipoprotein E epsilon 4 allele distributions in late-onset Alzheimer's disease and in other amyloid-forming diseases. Lancet 342:710-711

45. Shinohara M, Murray ME, Frank RD, Shinohara M, DeTure M, Yamazaki $Y$ et al (2016) Impact of sex and APOE4 on cerebral amyloid angiopathy in Alzheimer's disease. Acta Neuropathol 132:225-234

46. Thal DR, Rüb U, Orantes M, Braak H (2002) Phases of a beta-deposition in the human brain and its relevance for the development of $A D$. Neurology 58:1791-1800

47. Thal DR, Papassotiropoulos A, Saido TC, Griffin WS, Mrak RE, Kölsch $\mathrm{H}$ et al (2010) Capillary cerebral amyloid angiopathy identifies a distinct APOE epsilon4-associated subtype of sporadic Alzheimer's disease. Acta Neuropathol 120:169-183

48. Weller RO, Boche D, Nicoll JA (2009) Microvasculature changes and cerebral amyloid angiopathy in Alzheimer's disease and their potential impact on therapy. Acta Neuropathol 118:87-102

49. Wenham PR, Price WH, Blandell G (1991) Apolipoprotein E genotyping by one-stage PCR. Lancet 337:1158-1159

50. Wisniewski KE, Wisniewski HM, Wen GY (1985) Occurrence of neuropathological changes and dementia of Alzheimer's disease in Down's syndrome. Ann Neurol 17:278-282

51. Zigman WB, Jenkins EC, Tycko B, Schupf N, Silverman W (2005) Mortality is associated with apolipoprotein E $\varepsilon 4$ in non-demented adults with Down syndrome. Neurosci Lett 390:93-97

\section{Ready to submit your research? Choose BMC and benefit from:}

- fast, convenient online submission

- thorough peer review by experienced researchers in your field

- rapid publication on acceptance

- support for research data, including large and complex data types

- gold Open Access which fosters wider collaboration and increased citations

- maximum visibility for your research: over $100 \mathrm{M}$ website views per year

At BMC, research is always in progress.

Learn more biomedcentral.com/submissions 